\title{
Properties of $Z_{c}(\mathbf{3 9 0 0})$ tetraquark in a cold nuclear matter
}

\author{
K. Azizi $\oplus^{1,2}$ and N. $\mathrm{Er}^{3}$ \\ ${ }^{1}$ Department of Physics, University of Tehran, North Karegar Avenue, Tehran 14395-547, Iran \\ ${ }^{2}$ Department of Physics, Dogus University, Acibadem-Kadikoy, 34722 Istanbul, Turkey \\ ${ }^{3}$ Department of Physics, Abant İzzet Baysal University, Gölköy Kampüsü, 14980 Bolu, Turkey
}

(Received 25 January 2020; accepted 13 April 2020; published 27 April 2020)

\begin{abstract}
The study of medium effects on properties of particles embedded in nuclear matter is of great importance for understanding the nature and internal quark-gluon organization as well as exact determination of the quantum numbers, especially of the exotic states. In this context, we study the physical properties of one of the famous charmoniumlike states, $Z_{c}(3900)$, in a cold dense matter. We investigate the possible shifts in the mass and current-meson coupling of the $Z_{c}(3900)$ state due to the dense medium at saturation density $\rho^{\text {sat }}$ by means of the in-medium sum rules. We also estimate the vector self-energy of this state at saturation nuclear matter density. We discuss the behavior of the spectroscopic parameters of this state with respect to the density up to a high density corresponding to the core of neutron stars, $\rho \approx 5 \rho^{\text {sat }}$. Both the mass and current coupling of this state show nonlinear behavior and decrease with respect to the density of the medium: the mass reaches roughly $30 \%$ of its vacuum value at $\rho=5 \rho^{\text {sat }}$, while the current coupling approaches zero at $\rho \approx 2.1 \rho^{\text {sat }}$, when the central values of the auxiliary and other input parameters are used.
\end{abstract}

DOI: 10.1103/PhysRevD.101.074037

\section{INTRODUCTION}

The standard hadrons are divided into $q \bar{q}$ and $q q q / \bar{q} \bar{q} \bar{q}$ systems. Neither the quark model nor the QCD excludes the existence of the structures out of these configurations. Hence, search for exotic states is inevitable: now we have many exotic states observed in the experiment. We have also made good progress in the determination of different aspects of these states in theory. Most of the discovered exotic states are tetraquarks of the $X Y Z$ family. The term $X Y Z$ comes from the generalization of states $X(3872)$, $Y(4260)$, and $Z_{c}(3900)$. The $X Y Z$ states are the charmoniumlike or bottomoniumlike resonances, which, because of their mass, cannot be placed in the charmonium or bottomonium picture: These resonances, mainly with $Q \bar{Q} q \bar{q}$ quark content, have different properties than the standard excited quarkonium states. In the last decade, many $X Y Z$ states have been observed by the Belle, BESIII, $B A B A R$, LHCb, CMS, D0, CDF, and CLEO-c collaborations [1], and their masses, widths and quantum numbers $J^{P C}$ have been predicted. Detailed analysis of the experimental status of these states and various theoretical models can be found in numerous new review articles [2-5].

Published by the American Physical Society under the terms of the Creative Commons Attribution 4.0 International license. Further distribution of this work must maintain attribution to the author(s) and the published article's title, journal citation, and DOI. Funded by SCOAP .
In the first trials, the new charmoniumlike resonances, discovered in the above experiments, were evaluated as the excited states of ordinary $c \bar{c}$ charmonium states. However, the obtained data showed that some resonances do not conform to standard spectroscopy, and thus some new nonconventional models have been developed. The new models differ from each other in terms of their components and strong interaction mechanisms. There are plenty of studies for the physical picture interpretation of the charmoniumlike states using the new models: for instance, QCD tetraquarks [6,7], weakly bound hadronic molecules [8-14], charmonium hybrids [15-19], threshold cups [2023], and hadro-charmonium [24,25]. Understanding the nonperturbative behavior of QCD and the strong interaction dynamics that cause the production and structure of these nonconventional states is very important for today's experimental and theoretical studies.

As candidates of tetraquark states, $Z_{c}^{ \pm}(3900)$ were reported simultaneously by the BESIII Collaboration [26] using $e^{+} e^{-}$annihilation at the vector resonance $Y(4260)$ and by the Belle Collaboration [27] using the same process but at or near the $\Upsilon(n S)(n=1,2, \ldots, 5)$ resonances. For the full history, see [28-30]. They were confirmed by the CLEO-c Collaboration using $586 \mathrm{pb}^{-1}$ of $e^{+} e^{-}$annihilation data taken at the CESR collider at $\sqrt{s}=4170 \mathrm{MeV}$, the peak of the charmonium resonance $\psi(4160)$. They also reported evidence for $Z_{c}^{0}(3900)$, which is the neutral member of this isospin triplet [31]. In Ref. [32] $e^{+} e^{-} \rightarrow \pi^{ \pm}\left(D \bar{D}^{*}\right)^{\mp}$ at $\sqrt{s}=4.26 \mathrm{GeV}$ using $525 \mathrm{pb}^{-1}$ of data collected at the BEPCII storage ring, the 
determined pole mass $M_{\text {pole }}=(3883.9 \pm 1.5($ stat $) \pm$ 4.2(syst) $) \mathrm{MeV} / \mathrm{c}^{2}$, and pole width $\Gamma_{\text {pole }}=(24.8 \pm$ 3.3 (stat) \pm 11.0 (syst) $) \mathrm{MeV}$ were reported with a significance of $2 \sigma$ and $1 \sigma$, respectively. It was referred to as $Z_{c}(3885)$. BESIII also observed a new neutral state $Z_{c}(3900)^{0}$ in a process $e^{+} e^{-} \rightarrow \pi^{0} \pi^{0} J / \psi$ with a significance of $10.4 \sigma$. The measured mass and width were $(3894.8 \pm$ 2.3 (stat) \pm 3.2 (syst) $) \mathrm{MeV} / c^{2}$ and $\Gamma=(29.6 \pm 8.2$ (stat $) \pm$ 8.2(syst)) MeV, respectively [33]. In Ref. [34], after the full construction of the $D$ meson pair and the bachelor $\pi^{ \pm}$in the final state, the existence of the charged structure $Z_{c}^{ \pm}(3885)$ was confirmed in the $\left(D \bar{D}^{*}\right)^{\mp}$ system, and its pole mass and width were measured as $M_{\text {pole }}=(3881.7 \pm 1.6$ (stat $) \pm$ 1.6 (syst) $) \mathrm{MeV} / c^{2} \quad$ and $\quad \Gamma_{\text {pole }}=(26.6 \pm 2.0$ (stat $) \pm$ 2.1 (syst)) $\mathrm{MeV}$, respectively. In the processes $e^{+} e^{-} \rightarrow$ $D^{+} D^{*-} \pi^{0}+$ c.c. and $e^{+} e^{-} \rightarrow D^{0} \bar{D}^{* 0} \pi^{0}+$ c.c. at $\sqrt{s}=$ 4.226 and $4.257 \mathrm{GeV}$, the neutral structure $Z_{c}(3885)^{0}$ was observed with the pole mass $\left(3885.7_{-5.7}^{+4.3}\right.$ (stat $) \pm$ 8.4 (syst) $) \mathrm{MeV} / c^{2}$ and pole width $\left(35_{-12}^{+11}\right.$ (stat $) \pm$ 15 (syst)) $\mathrm{MeV}$ [35]. In a very recent study by the D0 Collaboration, the authors presented evidence for the $Z_{c}^{ \pm}(3900)$ state decaying to $J / \psi \pi^{ \pm}$in semi-inclusive weak decays of $b$-flavored hadrons [36].

On the theoretical side, for investigation of the $Z_{c}(3900)$ resonance, many different models and approaches are used; some of them are mentioned here as examples. In a recent study [37], using the three-channel Ross-Shaw theory, the authors have obtained constraint conditions that need to be satisfied by various parameters of the theory in order to have a narrow resonance close to the threshold of the third channel; this is relevant to the structure. Using the QCD sum rule method, the same state was considered as a compact tetraquark state of a diquark-antidiquark configuration in [38-40] and a hadronic molecule in [41,42]. In these studies, many parameters related to the $Z_{c}(3900)$ state were calculated. Its mass was already calculated within the framework of a nonrelativistic quark model in [43] as well.

Despite a lot of theoretical and experimental efforts, unfortunately, the nature and internal structures of most of the exotic states including $Z_{c}(3900)$ are not exactly clear. Hence, investigation of their properties at a dense or hot medium can play an important role. Experiments like PANDA will provide a possibility to explore these states in a dense medium. These studies will help us better understand the quark-gluon organization of the exotic states as well as their interactions with the particles existing in the medium. In our previous study, Ref. [44], we investigated the $X(3872)$ state by applying a diquark-antidiquark-type current in the framework of in-medium QCD sum rules. We calculated the mass, current-meson coupling, and also the vector self-energy of this state and found that these parameters strongly depend on the density of the medium. In the present study, we investigate the effects of a dense medium on the parameters of the $Z_{c}(3900)$ state and look for the behavior of the mass, current-meson coupling, and vector self-energy of this charmoniumlike state, considering it as a compact tetraquark state.

The study is organized as follows. In Sec. II, we derive the spectral densities associated with the state $Z_{c}(3900)$ by applying the two-point sum rule technique, and we obtain the QCD sum rules for the mass, current-meson coupling, and vector self-energy using the obtained spectral densities. In Sec. III, after fixing the auxiliary parameters using the standard prescriptions of the method, the numerical analysis of the physical observables, both in vacuum and cold nuclear matter, is performed. Section IV is devoted to a discussion and concluding remarks. We collect some lengthy expressions obtained from the calculations in the Appendix.

\section{IN-MEDIUM MASS AND CURRENT COUPLINGS OF $Z_{c}(3900)$}

Hadrons are formed as a result of some nonperturbative effects at low energies very far from the asymptotic region of QCD. Hence, to investigate their properties, some nonperturbative methods are needed. QCD sum rules appear as a reliable, powerful, and predictive approach in this respect. In this approach, the hadrons are represented by interpolating currents, written by considering the quark content and all the quantum numbers of the hadrons. From the theoretical studies in vacuum and the experimental data, the quantum numbers $J^{P C}=1^{+-}$have been assigned to $Z_{c}(3900)$. The comparison of the theoretical predictions on some parameters of this state with the experimental data leads us to consider a compact tetraquark of a diquark and an antidiquark structure for this state $[39,40]$. Thus, the interpolating current representing the $\bar{c} c u \bar{d}$ quark content and $J^{P C}=1^{+-}$quantum numbers can be written as

$$
\begin{aligned}
J_{\mu}(x)= & \frac{i \epsilon_{a b c} \epsilon_{d e c}}{\sqrt{2}}\left\{\left[u_{a}^{T}(x) C \gamma_{5} c_{b}(x)\right]\left[\bar{d}_{d}(x) \gamma_{\mu} C \bar{c}_{e}^{T}(x)\right]\right. \\
& \left.-\left[u_{a}^{T}(x) C \gamma_{\mu} c_{b}(x)\right]\left[\bar{d}_{d}(x) \gamma_{5} C \bar{c}_{e}^{T}(x)\right]\right\},
\end{aligned}
$$

where $\epsilon_{a b c}$ and $\epsilon_{d e c}$ are antisymmetric Levi-Civita symbols in three dimensions with color indices $a, b, c, d$, and $e$. The letter $T$ represents a transpose in Dirac space, $\gamma_{5}$ and $\gamma_{\mu}$ are Dirac matrices, and $C$ is the charge conjugation operator.

In the framework of QCD sum rules, we aim to obtain the sum rules for the mass, current coupling, and vector self-energy of the exotic state $Z_{c}$ in cold nuclear matter. In the generalization of the vacuum QCD sum rules to finite density, we start with the same correlation function of interpolating currents as the vacuum with the difference that, in this case, the time ordering product of the interpolating currents is sandwiched between the ground states of a finite density medium instead of vacuum [45]. So, we start with the following in-medium two-point correlation function as the building block of the method: 


$$
\Pi_{\mu \nu}(p)=i \int d^{4} x e^{i p \cdot x}\left\langle\psi_{0}\left|\mathcal{T}\left[J_{\mu}(x) J_{\nu}^{\dagger}(0)\right]\right| \psi_{0}\right\rangle
$$

where $p$ is the four-momentum of the $Z_{c}$ state, $\mathcal{T}$ is the time ordering operator, and $\left|\psi_{0}\right\rangle$ is the parity and time-reversal symmetric ground state of nuclear matter.

In cold nuclear matter, we formulate the sum rules for the modified mass, $m_{Z_{c}}^{*}$, and current coupling constant, $f_{Z_{c}}^{*}$, of the ground state $Z_{c}$. For this purpose and in accordance with the philosophy of the method, the correlation function is calculated in two different perspectives: the phenomenological (Phe) and operator product expansion (OPE) windows. The results of these two representations are then matched under some conditions like the quark-hadron duality assumption to relate the hadronic parameters to fundamental QCD parameters. We focus on the ground state; hence, we should apply some transformations like Borel and continuum subtraction to enhance the ground-state contribution and suppress the contributions coming from the higher states and continuum.

\section{A. Phenomenological window}

The phenomenological side of the correlation function is determined in a few steps: First, it is saturated by a full set of hadronic states carrying the same numbers as the interpolating current, and then the contribution of the ground state is isolated. As a result, we get

$$
\Pi_{\mu \nu}^{\mathrm{Phe}}(p)=-\frac{\left\langle\psi_{0}\left|J_{\mu}\right| Z_{c}(p)\right\rangle\left\langle Z_{c}(p)\left|J_{\nu}^{\dagger}\right| \psi_{0}\right\rangle}{p^{* 2}-m_{Z_{c}}^{* 2}}+\cdots,
$$

where $p^{*}$ is the in-medium momentum and dots denote contributions arising from higher resonances and continuum states. The decay constant or current-meson coupling is expressed in terms of the polarization vector $\varepsilon_{\mu}$ of $Z_{c}$ as

$$
\left\langle\psi_{0}\left|J_{\mu}\right| Z_{c}(p)\right\rangle=f_{Z_{c}}^{*} m_{Z_{c}}^{*} \varepsilon_{\mu},
$$

which further simplifies Eq. (3). By summing over the polarization vectors, we can recast the phenomenological side of the correlation function into the form

$$
\Pi_{\mu \nu}^{\mathrm{Phe}}(p)=-\frac{m_{Z_{c}}^{* 2} f_{Z_{c}}^{* 2}}{p^{* 2}-m_{Z_{c}}^{* 2}}\left[-g_{\mu \nu}+\frac{p_{\mu}^{*} p_{\nu}^{*}}{m_{Z_{c}}^{* 2}}\right]+\cdots
$$

To proceed, we introduce two self-energies: the scalar selfenergy $\Sigma_{s}=m_{Z_{c}}^{*}-m_{Z_{c}}$ and the vector self-energy $\Sigma_{v}$, which appears in the expression of the in-medium momentum, $p_{\mu}^{*}=p_{\mu}-\Sigma_{v} u_{\mu}$ [46], where $u_{\mu}$ is the four-velocity vector of the cold nuclear medium. We work in the rest frame of the medium, i.e., $u_{\mu}=(1,0)$. As a result, one can write

$$
\begin{aligned}
\Pi_{\mu \nu}^{\text {Phe }}(p)= & -\frac{f_{Z_{c}}^{* 2}}{p^{2}-\mu^{2}}\left[-g_{\mu \nu} m_{Z_{c}}^{* 2}+p_{\mu} p_{\nu}\right. \\
& \left.-\Sigma_{v} p_{\mu} u_{\nu}-\Sigma_{\nu} p_{\nu} u_{\mu}+\Sigma_{v}^{2} u_{\mu} u_{\nu}\right]+\cdots,
\end{aligned}
$$

where $\mu^{2}=m_{Z_{c}}^{* 2}-\Sigma_{v}^{2}+2 p_{0} \Sigma_{v}$, with $p_{0}=p$.u being the energy of the quasiparticle. The Borel transformed form (Borel with respect to $p^{2}$ ) of the phenomenological representation reads

$$
\begin{aligned}
\Pi_{\mu \nu}^{\mathrm{Phe}}(p)= & f_{Z_{c}}^{* 2} e^{-\mu^{2} / M^{2}}\left[-g_{\mu \nu} m_{Z_{c}}^{* 2}\right. \\
& \left.+p_{\mu} p_{\nu}-\Sigma_{\nu} p_{\mu} u_{\nu}-\Sigma_{\nu} p_{\nu} u_{\mu}+\Sigma_{v}^{2} u_{\mu} u_{\nu}\right] \\
& +\cdots,
\end{aligned}
$$

where $M^{2}$ is the Borel mass parameter to be fixed later using the standard recipe of the method.

\section{B. OPE or QCD window}

The QCD side of the calculations can be obtained by inserting the interpolating current $J_{\mu}(x)$ into the correlation function and performing all possible contractions of the quark pairs. The resultant equation is a expression in terms of the in-medium light quark $\left(S_{q}^{i j}\right)$ and heavy quark $\left(S_{Q}^{i j}\right)$ propagators:

$$
\begin{aligned}
\Pi_{\mu \nu}^{\mathrm{QCD}}(p)= & -\frac{i}{2} \varepsilon_{a b c} \varepsilon_{a^{\prime} b^{\prime} c^{\prime}} \varepsilon_{d e c} \varepsilon_{d^{\prime} e^{\prime} c^{\prime}} \int d^{4} x e^{i p x}\left\{\operatorname{Tr}\left[\gamma_{5} \tilde{S}_{u}^{a a^{\prime}}(x) \gamma_{5} S_{c}^{b b^{\prime}}(x)\right] \operatorname{Tr}\left[\gamma_{\mu} \tilde{S}_{c}^{e^{\prime} e}(-x) \gamma_{\nu} S_{d}^{d^{\prime} d}(-x)\right]-\operatorname{Tr}\left[\left[\gamma_{\mu} \tilde{S}_{c}^{e^{\prime} e}(-x)\right.\right.\right. \\
& \left.\times \gamma_{5} S_{d}^{d^{\prime} d}(-x)\right] \operatorname{Tr}\left[\gamma_{\nu} \tilde{S}_{u}^{a a^{\prime}}(x) \gamma_{5} S_{c}^{b b^{\prime}}(x)\right]-\operatorname{Tr}\left[\gamma_{5} \tilde{S}_{u}^{a a^{\prime}}(x) \gamma_{\mu} S_{c}^{b b^{\prime}}(x)\right] \operatorname{Tr}\left[\gamma_{5} \tilde{S}_{c}^{e^{\prime} e}(-x) \gamma_{\nu} S_{d}^{d^{\prime} d}(-x)\right] \\
& \left.+\operatorname{Tr}\left[\gamma_{\nu} \tilde{S}_{u}^{a a^{\prime}}(x) \gamma_{\mu} S_{c}^{b b^{\prime}}(x)\right] \operatorname{Tr}\left[\gamma_{5} \tilde{S}_{c}^{e^{\prime e}}(-x) \gamma_{5} S_{d}^{d^{\prime} d}(-x)\right]\right\}_{\left|\psi_{0}\right\rangle},
\end{aligned}
$$

where $\tilde{S}_{q(c)}=C S_{q(c)}^{T} C$.

The in-medium light and heavy quark propagators in coordinate space in the fixed point gauge and $m_{q} \rightarrow 0$ limit are given as 


$$
\begin{aligned}
S_{q}^{i j}(x)= & \frac{i}{2 \pi^{2}} \delta^{i j} \frac{1}{\left(x^{2}\right)^{2}} \not x+\chi_{q}^{i}(x) \bar{\chi}_{q}^{j}(0) \\
& -\frac{i g_{s}}{32 \pi^{2}} F_{\mu \nu}^{i j}(0) \frac{1}{x^{2}}\left[\not x \sigma^{\mu \nu}+\sigma^{\mu \nu} \not x\right]+\cdots,
\end{aligned}
$$

and

$$
\begin{aligned}
S_{c}^{i j}(x)= & \frac{i}{(2 \pi)^{4}} \int d^{4} k e^{-i k \cdot x}\left\{\frac{\delta_{i j}}{\not k-m_{c}}\right. \\
& -\frac{g_{s} F_{\mu \nu}^{i j}(0)}{4} \frac{\sigma_{\mu \nu}\left(\not k+m_{c}\right)+\left(\not k+m_{c}\right) \sigma_{\mu \nu}}{\left(k^{2}-m_{c}^{2}\right)^{2}} \\
& \left.+\frac{\pi^{2}}{3}\left\langle\frac{\alpha_{s} G G}{\pi}\right\rangle \delta_{i j} m_{c} \frac{k^{2}+m_{c} \not k}{\left(k^{2}-m_{c}^{2}\right)^{4}}+\cdots\right\} .
\end{aligned}
$$

In the above equations, $\chi_{q}^{i}$ and $\bar{\chi}_{q}^{j}$ are the Grassmann background quark fields and

$$
F_{\mu \nu}^{i j}=F_{\mu \nu}^{A} t^{i j, A}, \quad A=1,2, \ldots, 8,
$$

where $F_{\mu \nu}^{A}$ are classical background gluon fields, and $t^{i j, A}=\frac{\lambda^{i j, A}}{2}$, with $\lambda^{i j, A}$ being the standard Gell-Mann matrices. The next step is to use the expressions of the quark propagators in Eq. (8). This leads to two main contributions: perturbative and nonperturbative. The perturbative contributions, which represent the short distance effects, are calculated directly by applying the Fourier, Borel, and continuum subtraction procedures. The nonperturbative or long distance contributions are expressed in terms of the inmedium quark, gluon, and mixed condensates. For the explicit expressions of the condensates and the details of their expansions in terms of different operators, see, for instance, Ref. [44]. After insertion of the in-medium condensates in terms of various operators, the Fourier and Borel transformations as well as continuum subtraction are also applied to the nonperturbative part of the QCD side.

The QCD side of the correlation function can be decomposed over the selected Lorentz structures as

$$
\begin{aligned}
\Pi_{\mu \nu}^{\mathrm{QCD}}(p)= & -\Upsilon_{1}^{\mathrm{QCD}}\left(p^{2}\right) g_{\mu \nu}+\Upsilon_{2}^{\mathrm{QCD}}\left(p^{2}\right) p_{\mu} p_{\nu} \\
& -\Upsilon_{3}^{\mathrm{QCD}}\left(p^{2}\right) p_{\mu} u_{\nu}-\Upsilon_{4}^{\mathrm{QCD}}\left(p^{2}\right) p_{\nu} u_{\mu} \\
& +\Upsilon_{5}^{\mathrm{QCD}}\left(p^{2}\right) u_{\mu} u_{\nu},
\end{aligned}
$$

where the invariant functions $\Upsilon_{i}^{\mathrm{QCD}}(i=1, \ldots, 5)$ in Eq. (12) are given in terms of the following dispersion integrals:

$$
\Upsilon_{i}^{\mathrm{QCD}}\left(p^{2}\right)=\int_{4 m_{c}^{2}}^{\infty} \frac{\rho_{i}^{\mathrm{QCD}}(s)}{s-p^{2}} d s,
$$

where $\rho_{i}^{\mathrm{QCD}}(s)$ are the two-point spectral densities related to the imaginary parts of the selected coefficients. The main purpose of the QCD side of the calculations is to calculate these spectral densities. To this end and after insertion of the quark propagators into the correlation function, we use the relation

$$
\begin{aligned}
\frac{1}{\left(x^{2}\right)^{m}}= & \int \frac{d^{D} k}{(2 \pi)^{D}} e^{-i k \cdot x} i(-1)^{m+1} 2^{D-2 m} \pi^{D / 2} \\
& \times \frac{\Gamma[D / 2-m]}{\Gamma[m]}\left(-\frac{1}{k^{2}}\right)^{D / 2-m},
\end{aligned}
$$

to bring $x$ to the exponential. In this way, the resultant expressions contain four four-integrals: one over four- $k$ coming from the above relation, one initially existing in the correlation function (integral over four- $x$ ), and the last two over four- $k_{1}$ and four- $k_{2}$ coming from the heavy quark propagators. The next step is to perform the four-integral over $x$, which gives a Dirac delta function. We use this function to perform an integral over $k_{1}$. The remaining two integrals over four- $k_{2}$ and four- $k$ are performed using the Feynman parametrization and the formula

$$
\int d^{4} \ell \frac{\left(\ell^{2}\right)^{m}}{\left(\ell^{2}+\Delta\right)^{n}}=\frac{i \pi^{2}(-1)^{m-n} \Gamma[m+2] \Gamma[n-m-2]}{\Gamma[2] \Gamma[n](-\Delta)^{n-m-2}} .
$$

Finally, by applying the relation

$\Gamma\left[\frac{D}{2}-n\right]\left(-\frac{1}{\Delta}\right)^{D / 2-n}=\frac{(-1)^{n-1}}{(n-2) !}(-\Delta)^{n-2} \ln [-\Delta]$,

and using the expansion of $\ln [-\Delta]$, we get the imaginary parts of the $\Upsilon_{i}^{\mathrm{QCD}}$ functions.

We apply the Borel transformation with respect to the variable $p^{2}$ and perform the subtraction procedure according to the standard prescriptions of the method. As a result, we get

$$
\Upsilon_{i}^{\mathrm{QCD}}\left(M^{2}, s_{0}^{*}\right)=\int_{4 m_{c}^{2}}^{s_{0}^{*}} d s \rho_{i}^{\mathrm{QCD}}(s) e^{-\frac{s}{M^{2}}},
$$

where $s_{0}^{*}$ is the in-medium continuum threshold parameter separating the contributions of the ground state $Z_{c}$ and higher resonances and continuum. It will be fixed later. The spectral density related to each structure is the sum of the spectral densities of the perturbative (pert), two-quark ( $q q)$, two-gluon $(g g)$, and mixed quark-gluon ( $q g q)$ parts:

$$
\rho_{i}^{\mathrm{QCD}}(s)=\rho_{i}^{\mathrm{pert}}(s)+\rho_{i}^{q q}(s)+\rho_{i}^{g g}(s)+\rho_{i}^{q g q}(s) .
$$

As an example, for the $g_{\mu \nu}$ structure, these spectral densities are collected in the Appendix.

To obtain the QCD sum rules for the mass, current coupling constant, and vector self-energy of the $Z_{c}$ state, the coefficients of the same structures from both the $\Pi_{i}^{\text {Phe }}$ 
and $\Pi_{i}^{\mathrm{QCD}}$ functions are equated. We get the following sum rules for the physical quantities under consideration:

$$
\begin{aligned}
-m_{Z_{c}}^{* 2} f_{Z_{c}}^{* 2} e^{-\frac{\mu^{2}}{M^{2}}} & =\Upsilon_{1}^{\mathrm{QCD}}\left(M^{2}, s_{0}^{*}\right), \\
f_{Z_{c}}^{* 2} e^{-\frac{\mu^{2}}{M^{2}}} & =\Upsilon_{2}^{\mathrm{QCD}}\left(M^{2}, s_{0}^{*}\right), \\
-\Sigma_{v} f_{Z_{c}}^{* 2} e^{-\frac{\mu^{2}}{M^{2}}} & =\Upsilon_{3}^{\mathrm{QCD}}\left(M^{2}, s_{0}^{*}\right), \\
-\Sigma_{v} f_{Z_{c}}^{* 2} e^{-\frac{\mu^{2}}{M^{2}}} & =\Upsilon_{4}^{\mathrm{QCD}}\left(M^{2}, s_{0}^{*}\right), \\
\Sigma_{v}^{2} f_{Z_{c}}^{* 2} e^{-\frac{\mu^{2}}{M^{2}}} & =\Upsilon_{5}^{\mathrm{QCD}}\left(M^{2}, s_{0}^{*}\right) .
\end{aligned}
$$

\section{NUMERICAL RESULTS}

In this section, the QCD sum rules presented in Eq. (19) are used to investigate the behavior of the mass, current-meson coupling, and vector self-energy of the $Z_{c}$ state in cold nuclear matter. To this end, we need the values of some input parameters and in-medium condensates entering the expressions of the obtained sum rules. The in-medium expectation values of different operators, together with some other input parameters used in the calculations, are as follows: $\rho^{\text {sat }}=$ $0.11^{3} \mathrm{GeV}^{3}, \quad p_{0}=3887.2 \pm 2.3 \mathrm{MeV}[1],\left\langle q^{\dagger} q\right\rangle_{\rho}=\frac{3}{2} \rho$, $\langle\bar{q} q\rangle_{0}=(-0.241)^{3} \mathrm{GeV}^{3}$ [47], $\langle\bar{q} q\rangle_{\rho}=\langle\bar{q} q\rangle_{0}+\frac{\sigma_{\pi N}}{2 m_{q}} \rho$ [48], $m_{q}=\frac{m_{u}+m_{d}}{2}=0.00345 \mathrm{GeV} \quad[1], \quad\left\langle\frac{\alpha_{s}}{\pi} G^{2}\right\rangle_{0}=(0.33 \pm$ $0.04)^{4} \mathrm{GeV}^{4},\left\langle\frac{\alpha_{s}}{\pi} G^{2}\right\rangle_{\rho}=\left\langle\frac{\alpha_{s}}{\pi} G^{2}\right\rangle_{0}-(0.65 \pm 0.15) \mathrm{GeV} \rho$, $\left\langle q^{\dagger} i D_{0} q\right\rangle_{\rho}=0.18 \mathrm{GeV} \rho,\left\langle\bar{q} i D_{0} q\right\rangle_{\rho}=0,\left\langle q^{\dagger} i D_{0} i D_{0} q\right\rangle_{\rho_{N}}=$ $0.031 \mathrm{GeV}^{2} \rho_{N}-\frac{1}{12}\left\langle q^{\dagger} g_{s} \sigma G q\right\rangle_{\rho_{N}}, \quad\left\langle\bar{q} g_{s} \sigma G q\right\rangle_{0}=m_{0}^{2}\langle\bar{q} q\rangle_{0}$ [45], $\quad m_{0}^{2}=0.8 \mathrm{GeV}^{2}$ [47], $\left\langle\bar{q} g_{s} \sigma G q\right\rangle_{\rho}=\left\langle\bar{q} g_{s} \sigma G q\right\rangle_{0}+$ $3 \mathrm{GeV}^{2} \rho,\left\langle q^{\dagger} g_{s} \sigma G q\right\rangle_{\rho}=-0.33 \mathrm{GeV}^{2} \rho,\left\langle q^{\dagger} i D_{0} i D_{0} q\right\rangle_{\rho}=$ $0.031 \mathrm{GeV}^{2} \rho-\frac{1}{12}\left\langle q^{\dagger} g_{s} \sigma G q\right\rangle_{\rho}[45,48]$. The pion-nucleon sigma term $\sigma_{\pi N}=0.045 \mathrm{GeV}$ [49] is used. The quark masses are taken as $m_{u}=2.16_{-0.26}^{+0.49} \mathrm{MeV}, m_{d}=4.67_{-0.17}^{+0.48} \mathrm{MeV}$, and $m_{c}=1.27 \pm 0.02 \mathrm{GeV}[1]$.

Besides these inputs, the sum rules in Eqs. (19) require that we fix two auxiliary parameters: the Borel mass parameter $M^{2}$ and the in-medium continuum threshold $s_{0}^{*}$. To proceed, we need to determine their working regions, such that the in-medium mass, current coupling, and the vector self-energy show mild variations with respect to the changes in these parameters. The upper and lower limits for $M^{2}$ are determined via imposing some conditions according to the standard prescriptions of the method (for details in the case of doubly heavy baryons, see, for instance, Refs. [50,51]). The working window for $s_{0}^{*}$ is such that the maximum possible pole contribution is obtained, and the physical observables show weak dependence on the auxiliary parameters. These requirements lead to the working windows for auxiliary parameters displayed in Table I. As seen in Fig. 1, the average pole contribution changes in the interval [57\%-23\%], corresponding to $M^{2}=[3-5] \mathrm{GeV}^{2}$, which is reasonable in the case of tetraquarks. Our analyses
TABLE I. Working windows for $M^{2}$ and $s_{0}^{*}$.

\begin{tabular}{cc}
\hline \hline$M^{2}$ & {$[3-5] \mathrm{GeV}^{2}$} \\
$s_{0}^{*}$ & {$[17.6-19.4] \mathrm{GeV}^{2}$} \\
\hline \hline
\end{tabular}

show that the OPE series of sum rules converge very nicely within the working windows of the auxiliary parameters.

Prior to the investigation of the behavior of the inmedium physical quantities, we extract the vacuum mass value for the $Z_{c}$ state. The in-medium sum rules in the limit $\rho \rightarrow 0$ lead to the average value of vacuum mass as $\left(3932_{-84}^{+103}\right) \mathrm{MeV}$. We compare this value with the experimental data and other theoretical predictions in vacuum in Table II. As is seen, our prediction for the vacuum mass of $Z_{c}$ obtained via in-medium sum rules in the $\rho \rightarrow 0$ limit is consistent with other presented results within the errors arising mainly from the choice of the auxiliary parameters $M^{2}$ and $s_{0}^{*}$. Note that for Ref. [52], we presented only one of the results obtained via different scenarios. This result is the closest to the average experimental value.

Now, we proceed to display the behavior of physical quantities under consideration, with respect to the Borel mass and continuum threshold parameters at saturation nuclear matter density, $\rho^{\text {sat }}=0.11^{3} \mathrm{GeV}^{3}$. In this respect, we plot the ratio of the in-medium mass to vacuum mass, $m_{Z_{c}}^{*} / m_{Z_{c}}$, and the ratio of the in-medium current meson coupling to its vacuum value, $f_{Z_{c}}^{*} / f_{Z_{c}}$, in Fig. 2 . As it is clearly seen, the physical quantities show elegant stability against the changes in the parameters $M^{2}$ and $s_{0}^{*}$ in their working regions. At the saturation nuclear matter density, the in-medium mass of the $Z_{c}$ state decreases to approximately $77 \%$ of its vacuum mass, and the shift in the average current coupling value of the state due to nuclear medium is about $15 \%$. In Fig. 3, the quantities $m_{Z_{c}}^{*} / m_{Z_{c}}$ and $\Sigma_{\nu} / m_{Z_{c}}$ with respect to $M^{2}$ at the average value of the continuum threshold and saturation nuclear matter density

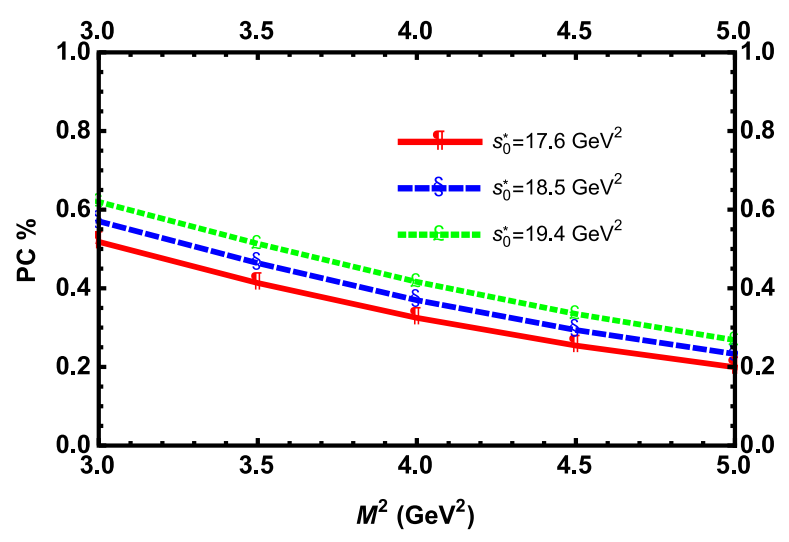

FIG. 1. The pole contribution in the $Z_{c}$ channel as a function of $M^{2}$ at saturation nuclear matter density and different fixed values of the in-medium continuum threshold. 
TABLE II. Experimental result and theoretical predictions of different methods for the vacuum mass of the $Z_{c}$ state. PS means present study, AAD means amplitude analysis of the data, and IMQCDSR refers to the in-medium QCD sum rules.

\begin{tabular}{lcl}
\hline \hline & \multicolumn{1}{c}{ Method } & \multicolumn{1}{c}{$m_{Z_{c}}$} \\
\hline PS & IMQCDSR $(\rho \rightarrow 0)$ & $\left(3932_{-84}^{+103}\right) \mathrm{MeV}$ \\
{$[1]$} & Experiment & $(3887.2 \pm 2.3) \mathrm{MeV}$ \\
{$[13]$} & QCDSR & $(3.88 \pm 0.17) \mathrm{GeV}$ \\
{$[53]$} & QCDSR & $\left(3.91_{-0.09}^{+0.11}\right) \mathrm{GeV}$ \\
{$[54]$} & QCDSR & $\left(3.89_{-0.09}^{+0.09}\right) \mathrm{GeV}$ \\
{$[52]$} & AAD & $\left(3893.2_{-7.7}^{+5.5}\right) \mathrm{MeV}$ \\
{$[40]$} & QCDSR & $\left(3901_{-148}^{+125}\right) \mathrm{MeV}$ \\
{$[19]$} & QCDSR & $(3.86 \pm 0.27) \mathrm{GeV}$ \\
\hline \hline
\end{tabular}

are shown. As already mentioned, the average negative shift in the modified mass of the $Z_{c}$ state due to cold nuclear matter (scalar self-energy) is about $23 \%$ of the vacuum mass, whereas the vector self-energy $\Sigma_{\nu}$ is obtained as approximately $32 \%$ of the vacuum value.

The main objective in the present study is to investigate the variations of the physical quantities with respect to the density of the nuclear medium. To this end, we plot in Fig. $4 m_{Z_{c}}^{*} / m_{Z_{c}}$ and $f_{Z_{c}}^{*} / f_{Z_{c}}$ as functions of $\rho / \rho^{\text {sat }}$ at

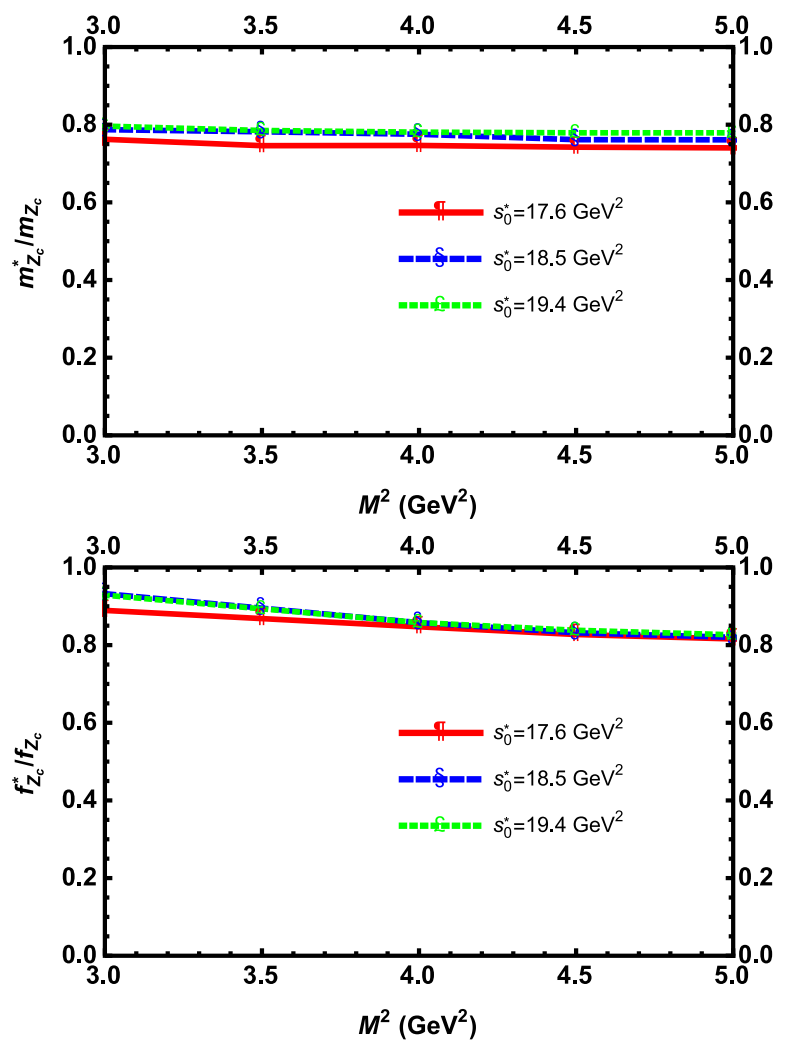

FIG. 2. The ratios $m_{Z_{c}}^{*} / m_{Z_{c}}$ and $f_{Z_{c}}^{*} / f_{Z_{c}}$ as functions of $M^{2}$ at the saturated nuclear matter density $\rho^{\text {sat }}=0.11^{3} \mathrm{GeV}^{3}$ and at fixed values of the continuum threshold.

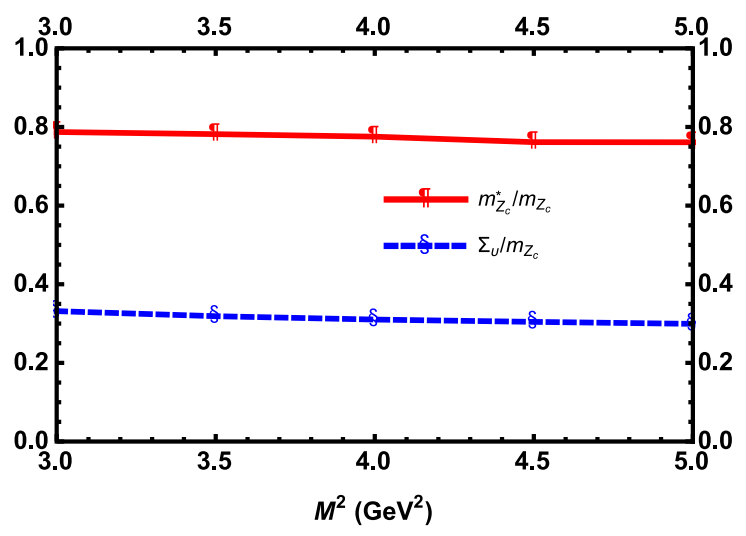

FIG. 3. The ratios $m_{Z_{c}}^{*} / m_{Z_{c}}$ and $\Sigma_{\nu} / m_{Z_{c}}$ as functions of $M^{2}$ at average values of the continuum threshold and at saturation nuclear matter density.

average values of the continuum threshold and Borel parameter. The saturation nuclear matter mass density is $\rho^{\text {sat }}=2.7 \times 10^{14} \mathrm{~g} / \mathrm{cm}^{3}$, which is equivalent to $\rho^{\text {sat }}=0.16 \mathrm{fm}^{-3}$. However, we need to know the behavior of hadrons at higher densities, which will be accessible in heavy ion collision experiments. Neutron stars as natural laboratories are very compact and dense and may produce hyperons and even heavy baryons and exotic states based on the processes that may occur inside them. For a neutron

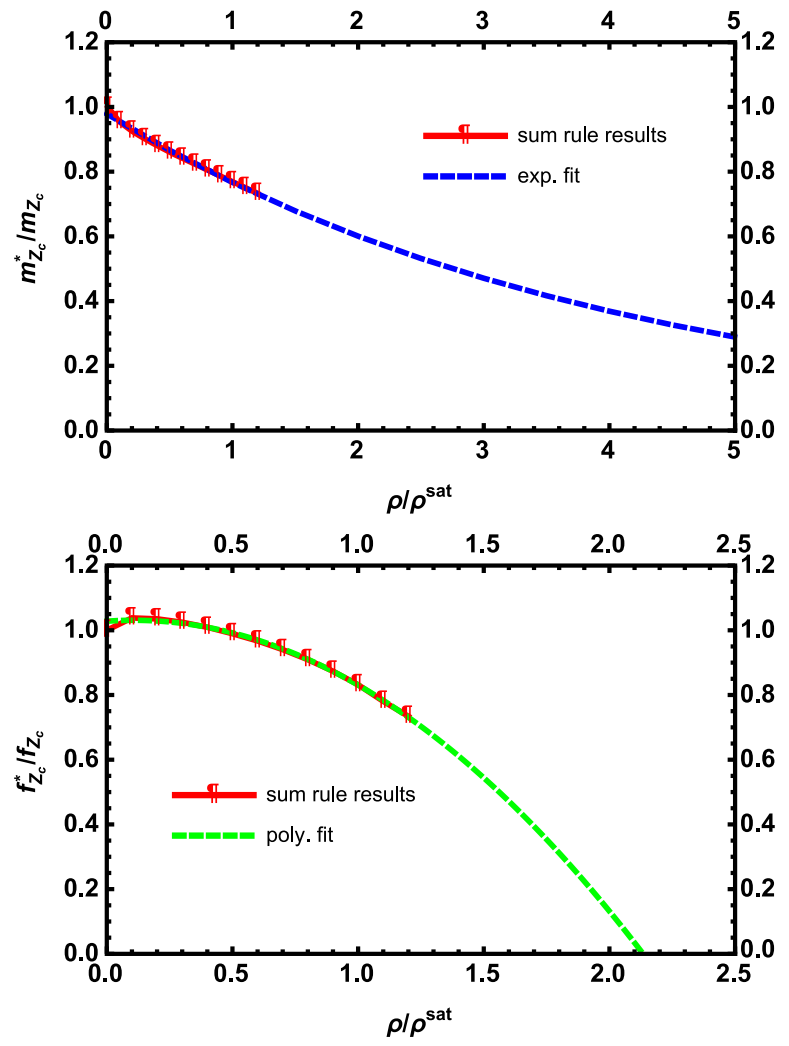

FIG. 4. The ratios $m_{Z_{c}}^{*} / m_{Z_{c}}$ and $f_{Z_{c}}^{*} / f_{Z_{c}}$ as functions of $\rho / \rho^{\text {sat }}$ at mean values of the continuum threshold and Borel parameter. 
star with mass $\sim 1.5 M_{\odot}$, the relevant core density is approximately $\left(2 \rho^{\text {sat }}-3 \rho^{\text {sat }}\right)$, and for the mass $\sim 2 M_{\odot}$ the same density is about $5 \rho^{\text {sat }}$ [55]. Therefore, we discuss the behavior of the mass and coupling constants up to densities comparable with the densities of the neutron stars. However, as is clear from Fig. 4, the in-medium sum rules give reliable results up to $\rho / \rho^{\text {sat }}=1$ and $\rho / \rho^{\text {sat }}=1.1$ for $m_{Z_{c}}^{*} / m_{Z_{c}}$ and $f_{Z_{c}}^{*} / f_{Z_{c}}$, respectively. Hence, we need to extrapolate the results to include the higher densities. Our analyses show that the following fit functions well describe the ratios under consideration when the central values of the auxiliary and other input parameters are used:

$$
m_{Z_{c}}^{*} / m_{Z_{c}}=e^{-0.252 x}
$$

and

$$
f_{Z_{c}}^{*} / f_{Z_{c}}=-0.251 x^{2}+0.054 x+1.028
$$

where $x=\rho / \rho^{\text {sat }}$. From Fig. 4, we see that the fit results coincide with the sum rule predictions at lower densities. The results show that the mass exponentially decreases with respect to $x$ and reaches roughly $30 \%$ of the vacuum mass at a density $5 \rho^{\text {sat }}$. The coupling constant, however, rapidly changes with respect to $x$ and goes to zero at $x=2.1$. This point may be considered as a pseudocritical density, at which hadrons are melted.

It is necessary to check the results at higher densities using different fit functions that may end up in different predictions. Among various fit functions, the best ones are as follows: exponential fit for the mass ratio and polynomial fit for the coupling constant ratio. Our analyses show that another set of fit functions, although not as good as the above selected functions, describe the behaviors of the quantities under study with respect to density as well. These are the $p$-pole fit for $m_{Z_{c}}^{*} / m_{Z_{c}}$ :

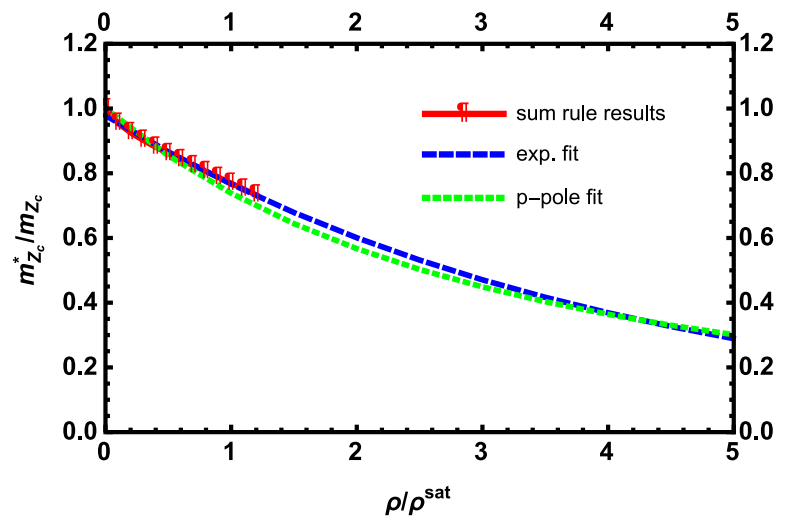

FIG. 5. Comparison between exponential and $p$-pole fit functions for $m_{Z_{c}}^{*} / m_{Z_{c}}$ changes with respect to $\rho / \rho^{\text {sat }}$.

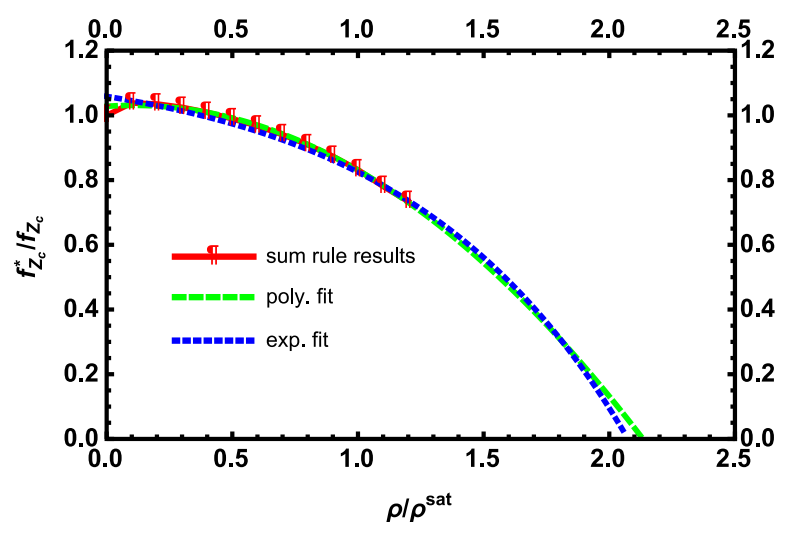

FIG. 6. Comparison between polynomial and exponential fit functions for $f_{Z_{c}}^{*} / f_{Z_{c}}$ changes with respect to $\rho / \rho^{\text {sat. }}$.

$$
\frac{m_{Z_{c}}^{*}}{m_{Z_{c}}}=\frac{1}{\left[1+\frac{x^{2}}{p a}\right]^{p}},
$$

where $p=2.035$ and $a=3.056$, and the exponential fit for $f_{Z_{c}}^{*} / f_{Z_{c}}$ :

$$
f_{Z_{c}}^{*} / f_{Z_{c}}=1.169-0.111 e^{-x / 0.879}
$$
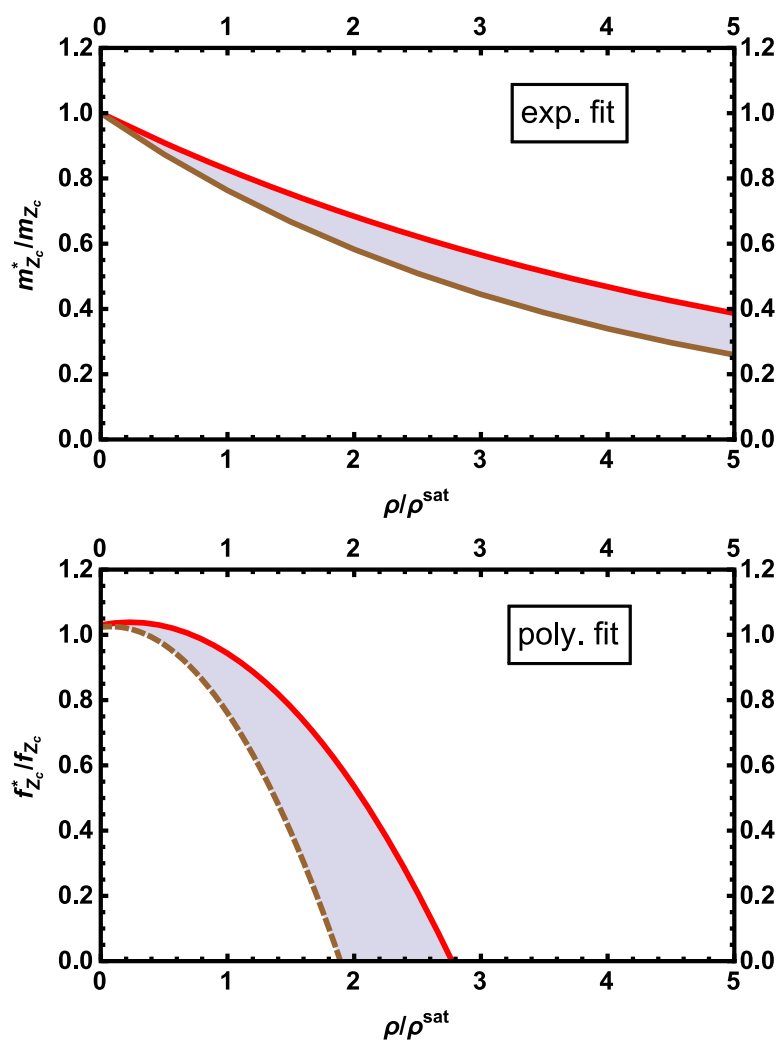

FIG. 7. The ratios $m_{Z_{c}}^{*} / m_{Z_{c}}$ and $f_{Z_{c}}^{*} / f_{Z_{c}}$ as functions of $\rho / \rho^{\text {sat }}$ when the uncertainties of the auxiliary and other input parameters are considered. 
In Figs. 5 and 6, we compare the predictions of these fit functions with the predictions of the previous fit functions. In the case of the mass ratio, although both fitting results show a similar behavior, the $p$-pole fitting leads to a result which is $6.4 \%$ larger than the exponential fitting result at the density $5 \rho^{\text {sat }}$. In the case of the coupling constant, $f_{Z_{c}}^{*} / f_{Z_{c}}$ becomes zero at $\rho / \rho^{\text {sat }}=2.13$ and $\rho / \rho^{\text {sat }}=2.07$, for the polynomial and exponential fittings, respectively. The small differences in the predictions of different fit functions remain inside the uncertainties allowed by the method used.

Now, we add the uncertainties in the values of the auxiliary parameters and errors of other inputs to the predictions at higher densities. For the case of best fits for the mass and coupling ratios, i.e., Eqs. (20) and (21), the swept areas presented in Fig. 7 are obtained. As we can see, the values of the ratio $m_{Z_{c}}^{*} / m_{Z_{c}}$ vary in the region [0.26, $0.39]$ at $\rho / \rho^{\text {sat }}=5$. The band of the coupling ratio $f_{Z_{c}}^{*} / f_{Z_{c}}$ becomes zero and crosses the $\rho / \rho^{\text {sat }}$ axis in the interval $[1.9,2.8]$.

\section{DISCUSSION AND CONCLUSION}

Despite much experimental and theoretical effort, the structure, quark-gluon organization, and nature of most exotic states remain unclear. The tetraquark state $Z_{c}(3900)$ is among the charmoniumlike resonances that deserve further investigation in vacuum and a dense or hot medium in order to fix its nature. We considered it as a compact tetraquark and assigned a diquark-antidiquark structure to it with quantum numbers $J^{P C}=1^{+-}$. We constructed inmedium sum rules to calculate its mass, current coupling, and vector self-energy in cold nuclear matter. The result obtained for its mass in the $\rho \rightarrow 0$ limit is compatible with the experimental data and vacuum theory predictions. At saturation density, the mass and current coupling receive negative shifts with respect to the vacuum values. These shifts amount to $23 \%$ and $15 \%$ for the mass and current coupling, respectively. This state receives a repulsive vector self-energy with an amount of $32 \%$ of its vacuum mass value at saturation nuclear matter density.

The in-medium experiments aim to reach higher densities. The neutron stars as compact and dense objects are natural laboratories with densities 2-5 times greater than the saturation nuclear matter density. The processes that occur at high densities may produce different kinds of hadrons, even exotic states. Experiments that take place at high densities will serve as a good opportunity to study the exotic states like $Z_{c}$. Hence, to provide some phenomenological predictions, we investigated the behavior of the state under consideration with respect to the density of the medium at higher densities. The in-medium sum rules are truncated at around $\rho / \rho^{\text {sat }}=(1-1.1)$. Thus, we used some fit functions in order to extrapolate the results to a density around $5 \rho^{\text {sat }}$. Our analyses show that the mass and current coupling constant exhibit nonlinear behavior and decrease with respect to density. The mass reaches roughly $30 \%$ of the vacuum mass at a density $5 \rho^{\text {sat }}$ if the central values of the auxiliary and other input parameters, as well as the best fit, are considered. When the uncertainties of the parameters are taken into account, this value lies in the interval $[26 \%, 39 \%]$. The current coupling, however, rapidly decreases and goes to zero at a density $\approx 2.1 \rho^{\text {sat }}$ if the central values of all parameters are considered, which may be considered as a pseudocritical density for melting the exotic charmoniumlike states. When the uncertainties in the values of the auxiliary and input parameters are taken into account, the band of $f_{Z_{c}}^{*} / f_{Z_{c}}$ becomes zero and crosses the $\rho / \rho^{\text {sat }}$ axis in the region $[1.9,2.8]$.

Investigation of properties of hadrons at finite temperature and density constitutes one of the main directions of research in high energy and nuclear physics. Understanding the hadronic behavior under extreme conditions can help us not only understand the internal structure and nature of hadrons and gain knowledge of the QCD as the theory of the strong interaction, but also analyze the results of future experiments as well as understand different possible phases of matter.

\section{ACKNOWLEDGMENTS}

The authors thank TUBITAK for the partial support provided under Grant No. 119F094.

\section{APPENDIX: SPECTRAL DENSITIES}

We present the explicit forms of the spectral densities for the $g_{\mu \nu}$ structure used in the calculations:

$$
\begin{aligned}
\rho_{1}^{\text {pert }}(s)= & -\frac{1}{3072 \pi^{6}} \int_{0}^{1} d z \int_{0}^{1-z} d w \frac{1}{\xi \vartheta^{8}}\left[w z \left[\left(s w z(w+z-1)-m_{c}^{2}\left[\left(w^{3}+w^{2}(2 z-1)\right.\right.\right.\right.\right. \\
& \left.\left.+2 w(z-1) z+(z-1) z^{2}\right]\right]^{2}\left[3 m_{c}^{4}\left[w^{3}+w^{2}(2 z-1)+2 w(z-1) z+(z-1) z^{2}\right]^{2}\right. \\
& -26 m_{c}^{2} s w z\left[w^{4}+w^{3}(3 z-2)+w^{2}\left(4 z^{2}-5 z+1\right)+w z\left(3 z^{2}-5 z+2\right)+(z-1)^{2} z^{2}\right] \\
& \left.+35 s^{2} w^{2} z^{2}(w+z-1)^{2}\right] \Theta[L(s, z, w)],
\end{aligned}
$$




$$
\begin{aligned}
& \rho_{1}^{q q}(s)=\frac{1}{4 \pi^{4}} \int_{0}^{1} d z \int_{0}^{1-z} d w\left\{\frac { m _ { c } w } { 4 \vartheta ^ { 5 } } \left[3 m_{c}^{4}\left[w^{3}+w^{2}(2 z-1)+2 w(z-1) z+(z-1) z^{2}\right]^{2}\right.\right. \\
& -10 m_{c}^{2} s w z\left[w^{4}+w^{3}(3 z-2)+w^{2}\left(4 z^{2}-5 z+1\right)+w z\left(3 z^{2}-5 z+2\right)+(z-1)^{2} z^{2}\right] \\
& \left.+7 s^{2} w^{2} z^{2}(w+z-1)^{2}\right]\langle\bar{u} u\rangle_{\rho}+\frac{m_{q} m_{c} p_{0} w^{2} z \xi}{\vartheta^{5}}\left[5 m _ { c } ^ { 2 } \left(w^{3}+w^{2}(2 z-1)\right.\right. \\
& \left.\left.+2 w(z-1) z+(z-1) z^{2}\right)-7 s w z \xi\right]\left\langle u^{\dagger} u\right\rangle_{\rho}+\frac{m_{c} z}{4 \vartheta^{5}}\left[3 m _ { c } ^ { 4 } \left[w^{3}+w^{2}(2 z-1)\right.\right. \\
& \left.+2 w(z-1) z+(z-1) z^{2}\right]^{2}-10 m_{c}^{2} s w z\left[w^{4}+w^{3}(3 z-2)+w^{2}\left(4 z^{2}-5 z+1\right)\right. \\
& \left.\left.+w z\left(3 z^{2}-5 z+2\right)+(z-1)^{2} z^{2}\right]+7 s^{2} w^{2} z^{2}(w+z-1)^{2}\right]\langle\bar{d} d\rangle_{\rho} \\
& +\frac{m_{q} m_{c} p_{0} w z^{2} \xi}{\vartheta^{5}}\left[5 m_{c}^{2}\left(w^{3}+w^{2}(2 z-1)+2 w(z-1) z+(z-1) z^{2}\right)-7 s w z \xi\right] \\
& \left.\times\left\langle d^{\dagger} d\right\rangle_{\rho}\right\} \Theta[L(s, z, w)] \\
& \rho_{1}^{g g}(s)=\frac{1}{96 \pi^{4}} \int_{0}^{1} d z \int_{0}^{1-z} d w\left\{\left[-i \frac{m_{q} m_{c}}{\vartheta^{4}}\left[m _ { c } ^ { 2 } \left(4 w^{6} z+w^{5}\left(24 z^{2}-8 z+1\right)\right.\right.\right.\right. \\
& +w^{4}\left(52 z^{3}-44 z^{2}+6 z-1\right)+w^{3} z\left(60 z^{3}-80 z^{2}+55 z-2\right)+w^{2} z^{2}\left(40 z^{3}-68 z^{2}\right. \\
& \left.+95 z-34)+6 w z^{3}\left(2 z^{3}-4 z^{2}+13 z-11\right)+33(z-1) z^{4}\right)+w z \xi\left(4 p _ { 0 } ^ { 2 } \left(4 w^{3} z\right.\right. \\
& \left.+w^{2}\left(16 z^{2}-4 z+1\right)+12 w(z-1) z^{2}+z^{2}\right)-3 s\left(4 w^{3} z+w^{2}\left(16 z^{2}-4 z+1\right)\right. \\
& \left.\left.\left.\left.+12 w(z-1) z^{2}+17 z^{2}\right)\right)\right]\right]\left\langle u^{\dagger} i D_{0} u\right\rangle_{\rho}+\frac{1}{1536(w-1) \xi^{2} \vartheta^{6}}\left[m _ { c } ^ { 4 } ( w - 1 ) \left(w^{2}\right.\right. \\
& +w(z-1)+(z-1) z)^{2}\left(24 w^{8} z+12 w^{7}\left(11 z^{2}-8 z+3\right)+3 w^{6}\left(112 z^{3}-156 z^{2}\right.\right. \\
& +45 z-36)+2 w^{5}\left(258 z^{4}-516 z^{3}+332 z^{2}-201 z+54\right)+w^{4}\left(516 z^{5}-1320 z^{4}+1574 z^{3}\right. \\
& \left.-1198 z^{2}+471 z-36\right)+w^{3} z\left(336 z^{5}-1032 z^{4}+1707 z^{3}-1769 z^{2}+858 z-132\right) \\
& +w^{2} z^{2}\left(132 z^{5}-468 z^{4}+1122 z^{3}-1253 z^{2}+807 z-180\right)+4 w z^{3}\left(6 z^{5}-24 z^{4}+152 z^{3}\right. \\
& \left.\left.-155 z^{2}+96 z-27\right)+8 z^{4}\left(23 z^{3}-29 z^{2}+9 z-3\right)\right)-m_{q}^{2} s(w-1) w z\left(24 w^{10} z\right. \\
& +12 w^{9}\left(13 z^{2}-12 z+3\right)+w^{8}\left(492 z^{3}-852 z^{2}-89 z-180\right)+w^{7}\left(984 z^{4}-2424 z^{3}+283 z^{2}\right. \\
& +672 z+360)+w^{6}\left(1368 z^{5}-4296 z^{4}+2149 z^{3}+1121 z^{2}-270 z-360\right)+w^{5}\left(1368 z^{6}\right. \\
& \left.-5160 z^{5}+4057 z^{4}-420 z^{3}+275 z^{2}-676 z+180\right)+w^{4}\left(984 z^{7}-4296 z^{6}+4151 z^{5}\right. \\
& \left.-809 z^{4}+1804 z^{3}-1901 z^{2}+615 z-36\right)+w^{3} z\left(492 z^{7}-2424 z^{6}+2485 z^{5}+834 z^{4}\right. \\
& \left.+43 z^{3}-2396 z^{2}+1098 z-132\right)+w^{2} z^{2}\left(156 z^{7}-852 z^{6}+898 z^{5}\right. \\
& \left.+1887 z^{4}-2785 z^{3}-27 z^{2}+903 z-180\right)+12 w(z-1)^{2} z^{3}\left(2 z^{5}-8 z^{4}+18 z^{3}+89 z^{2}\right. \\
& \left.-12 z-9)+8(z-1)^{3} z^{4}\left(23 z^{2}+30 z+3\right)\right)-12 s w z^{2}(w+z-1)^{3}\left(w^{5}((17 s+4) z-4)\right. \\
& +w^{4}\left(5(3 s+2) z^{2}-2(17 s+4) z+2\right)+w^{3} z\left(s\left(32 z^{2}-13 z+17\right)+4\left(3 z^{2}-4 z+1\right)\right) \\
& +2 w^{2} z^{2}\left(s\left(16 z^{2}-32 z-1\right)+7 z^{2}-10 z+3\right)-8 w(z-1) z^{3}(4 s-z+1)+2 w^{6} \\
& \left.\left.\left.+4(z-1)^{2} z^{4}\right)\right]\left\langle\frac{\alpha_{s}}{\pi} G^{2}\right\rangle_{\rho}\right\} \Theta[L(s, z, w)] \text {, }
\end{aligned}
$$

and

$$
\rho_{1}^{q g q}(s)=\int_{0}^{1} d z \int_{0}^{1-z} d w\left[\rho_{1,1}^{q g q}(s)+\rho_{1,2}^{q g q}(s)+\rho_{1,3}^{q g q}(s)+\rho_{1,4}^{q g q}(s)+\rho_{1,5}^{q g q}(s)\right] \Theta[L(s, z, w)],
$$


where

$$
\begin{aligned}
& \rho_{1,1}^{q g q}(s)=\frac{1}{96 \pi^{4}}\left[-\frac{m_{c}}{\kappa^{6}}\left[m _ { c } ^ { 2 } \left(4 w^{6} z+w^{5}\left(24 z^{2}-8 z+1\right)+w^{4}\left(52 z^{3}-44 z^{2}-2 z-1\right)+w^{3} z\right.\right.\right. \\
& \times\left(60 z^{3}-80 z^{2}+31 z+6\right)+w^{2} z^{2}\left(40 z^{3}-68 z^{2}+71 z-18\right)+2 w z^{3}\left(6 z^{3}-12 z^{2}\right. \\
& \left.+35 z-29)+33(z-1) z^{4}\right)\left(w^{2}+w(z-1)+(z-1) z\right)^{3}+w z(w+z-1)\left(4 p _ { 0 } ^ { 2 } \left(4 w^{9} z\right.\right. \\
& +w^{8}\left(28 z^{2}-16 z+1\right)+3 w^{7}\left(28 z^{3}-36 z^{2}+25 z-1\right)+w^{6}\left(160 z^{4}-300 z^{3}+371 z^{2}\right. \\
& -169 z+3)+w^{5}\left(208 z^{5}-508 z^{4}+838 z^{3}-694 z^{2}+157 z-1\right)+w^{4} z\left(192 z^{5}-564 z^{4}\right. \\
& \left.+1116 z^{3}-1276 z^{2}+567 z-51\right)+2 w^{3} z^{2}\left(62 z^{5}-210 z^{4}+463 z^{3}-642 z^{2}+409 z-82\right) \\
& +w^{2}(z-1)^{2} z^{3}\left(52 z^{3}-92 z^{2}+239 z-164\right)+3 w(z-1)^{3} z^{4}\left(4 z^{2}-4 z+17\right) \\
& \left.+(z-1)^{3} z^{5}\right)-s\left(12 w^{9} z+w^{8}\left(84 z^{2}-48 z+3\right)+w^{7}\left(252 z^{3}-324 z^{2}+121 z-9\right)\right. \\
& +w^{6}\left(480 z^{4}-900 z^{3}+713 z^{2}-195 z+9\right)+w^{5}\left(624 z^{5}-1524 z^{4}+1730 z^{3}-986 z^{2}\right. \\
& +159 z-3)+w^{4} z\left(576 z^{5}-1692 z^{4}+2500 z^{3}-2060 z^{2}+709 z-49\right)+2 w^{3} z^{2}\left(186 z^{5}\right. \\
& \left.-630 z^{4}+1125 z^{3}-1218 z^{2}+635 z-98\right)+w^{2}(z-1)^{2} z^{3}\left(156 z^{3}-276 z^{2}+589 z-292\right) \\
& \left.\left.\left.\left.+w(z-1)^{3} z^{4}\left(36 z^{2}-36 z+193\right)+51(z-1)^{3} z^{5}\right)\right)\right]\right]\left\langle\bar{u} i D_{0} i D_{0} u\right\rangle_{\rho}, \\
& \rho_{1,2}^{q g q}(s)=\frac{1}{12 \pi^{4}}\left[\frac { m _ { c } w z ^ { 2 } \xi } { \vartheta ^ { 7 } } \left[m_{c}^{2}(w+z)\left(w^{2}+w(z-1)+(z-1) z\right)^{3}-w z \xi\right.\right. \\
& \times\left(8 p _ { 0 } ^ { 2 } \left(3 w^{4}+w^{3}(7 z-6)+w^{2}(7 z-6)+w^{2}\left(10 z^{2}-14 z+3\right)+w z\left(6 z^{2}-13 z+7\right)\right.\right. \\
& \left.+3(z-1)^{2} z^{2}\right)-s\left(5 w^{4}+2 w^{3}(6 z-5)+w^{2}\left(17 z^{2}-24 z+5\right)+2 w z\left(5 z^{2}-11 z\right.\right. \\
& \left.\left.\left.\left.+6)+5(z-1)^{2} z^{2}\right)\right)\right]\right]\left\langle\bar{d} i D_{0} i D_{0} d\right\rangle_{\rho} \\
& \rho_{1,3}^{q g q}(s)=\frac{1}{96 \pi^{4}}\left[\frac { m _ { c } } { 4 \vartheta ^ { 7 } } \left[w z ( w + z - 1 ) \left(2 p _ { 0 } ^ { 2 } \left(4 w^{9} z+w^{8}\left(28 z^{2}-16 z+1\right)\right.\right.\right.\right. \\
& +3 w^{7}\left(28 z^{3}-36 z^{2}+25 z-1\right)+w^{6}\left(160 z^{4}-300 z^{3}+371 z^{2}\right. \\
& \left.-169 m_{c} z+3\right)+w^{5}\left(208 z^{5}-508 z^{4}+838 z^{3}-694 z^{2}+157 z-1\right)+w^{4} z \\
& \times\left(192 z^{5}-564 z^{4}+1116 z^{3}-1276 z^{2}+567 z-51\right)+2 w^{3} z^{2}\left(62 z^{5}-210 z^{4}\right. \\
& \left.+463 z^{3}-642 z^{2}+409 z-82\right)+w^{2}(z-1)^{2} z^{3}\left(52 z^{3}-92 z^{2}+239 z\right. \\
& \left.-164)+3 w(z-1)^{3} z^{4}\left(4 z^{2}-4 z+17\right)+(z-1)^{3} z^{5}\right)+s\left(4 w^{9} z+w^{8}\left(28 z^{2}\right.\right. \\
& -16 z-59)+w^{7}\left(84 z^{3}-108 z^{2}-401 z+177\right)+w^{6}\left(160 z^{4}-300 z^{3}-1197 z^{2}\right. \\
& +1259 z-177)+w^{5}\left(208 z^{5}-508 z^{4}-2022 z^{3}+3534 z^{2}-1271 z+59\right) \\
& +w^{4} z\left(192 z^{5}-564 z^{4}-2204 z^{3}+5368 z^{2}-3185 z+425\right)+2 w^{3} z^{2}\left(62 z^{5}-210 z^{4}\right. \\
& \left.-751 z^{3}+2380 z^{2}-1945 z+464\right)+w^{2}(z-1)^{2} z^{3}\left(52 z^{3}-92 z^{2}-861 z+760\right) \\
& \left.\left.+w(z-1)^{3} z^{4}\left(12 z^{2}-12 z-173\right)+25(z-1)^{3} z^{5}\right)\right)-m_{c}^{2}\left(w^{2}+w(z-1)\right. \\
& +(z-1) z)^{3}\left(4 w^{6} z+w^{5}\left(24 z^{2}-8 z-35\right)+w^{4}\left(52 z^{3}-44 z^{2}-170 z+35\right)+w^{3} z\left(60 z^{3}\right.\right. \\
& \left.-80 z^{2}-353 z+174\right)+w^{2} z^{2}\left(40 z^{3}-68 z^{2}-301 z+234\right)+2 w z^{3}\left(6 z^{3}-12 z^{2}-37 z\right. \\
& \left.\left.\left.+43)+9(z-1) z^{4}\right)\right]\right]\left\langle\bar{u} g_{s} \sigma G u\right\rangle_{\rho}
\end{aligned}
$$




$$
\begin{aligned}
\rho_{1,4}^{q g q}(s)= & \frac{1}{48 \pi^{4}}\left[\frac { m _ { c } w z ^ { 2 } \xi } { \vartheta ^ { 7 } } \left[13 m_{c}^{2}(w+z)\left(w^{2}+w(z-1)+(z-1) z\right)^{3}+w z \xi\right.\right. \\
& \times\left(4 p _ { 0 } ^ { 2 } \left(3 w^{4}+w^{3}(7 z-6)+w^{2}\left(10 z^{2}-14 z+3\right)+w z\left(6 z^{2}-13 z+7\right)\right.\right. \\
& \left.+3(z-1)^{2} z^{2}\right)-s\left(31 w^{4}+w^{3}(66 z-62)+w^{2}\left(97 z^{2}-132 z+31\right)+2 w z\left(31 z^{2}-64 z\right.\right. \\
& \left.\left.\left.\left.+33)+31(z-1)^{2} z^{2}\right)\right)\right]\right]\left\langle\bar{d} g_{s} \sigma G d\right\rangle_{\rho}
\end{aligned}
$$

and

$$
\begin{aligned}
\rho_{1,5}^{q g q}(s)= & \frac{1}{192 \pi^{4}}\left[\frac { p _ { 0 } z } { \vartheta ^ { 4 } } \left[m _ { c } ^ { 2 } \left(16 w^{7} z+w^{6}\left(48 z^{2}-48 z+31\right)+w^{5}\left(80 z^{3}-128 z^{2}+137 z-62\right)\right.\right.\right. \\
& +w^{4}\left(80 z^{4}-176 z^{3}+237 z^{2}-164 z+31\right)+w^{3} z\left(48 z+4-128 z^{3}+223 z^{2}-190 z+59\right) \\
& +w^{2} z^{2}\left(16 z^{4}-48 z^{3}+109 z^{2}-110 z+33\right)+w z^{3}\left(25 z^{2}-38 z+13\right) \\
& \left.\left.\left.+8(z-1)^{2} z^{4}\right)\right]\right]\left\langle u^{\dagger} g_{s} \sigma G u\right\rangle_{\rho}
\end{aligned}
$$

The shorthand notations in the above equations are given as

$$
\begin{aligned}
\xi & =(w+z-1) \\
\vartheta & =\left(w^{2}+w(z-1)+(z-1) z\right) \\
L[s, w, z] & =\frac{\left((-1+w)\left(-(s w z(-1+w+z))+m_{c}^{2}\left(w^{3}+2 w(-1+z) z+(-1+z) z^{2}+w^{2}(-1+2 z)\right)\right)\right)}{\left(w^{2}+w(-1+z)+(-1+z) z\right)^{2}} .
\end{aligned}
$$

[1] M. Tanabashi et al., Review of particle physics, Phys. Rev. D 98, 030001 (2018).

[2] Y.-R. Liu, H.-X. Chen, W. Chen, X. Liu, and S.-L. Zhu, Pentaquark and tetraquark states, Prog. Part. Nucl. Phys. 107, 237 (2019).

[3] F.-K. Guo, C. Hanhart, U.-G. Meißner, Q. Wang, Q. Zhao, and B.-S. Zou, Hadronic molecules, Rev. Mod. Phys. 90, 015004 (2018).

[4] A. Ali, J. S. Lange, and S. Stone, Exotics: Heavy pentaquarks and tetraquarks, Prog. Part. Nucl. Phys. 97, 123 (2017).

[5] S. L. Olsen, T. Skwarnicki, and D. Zieminska, Nonstandard heavy mesons and baryons: Experimental evidence, Rev. Mod. Phys. 90, 015003 (2018).

[6] P. C. Wallbott, G. Eichmann, and C. S. Fischer, X(3872) as a four-quark state in a Dyson-Schwinger/Bethe-Salpeter approach, Phys. Rev. D 100, 014033 (2019).

[7] S.-Q. Luo, K. Chen, X. Liu, Y.-R. Liu, and S.-L. Zhu, Exotic tetraquark states with the $q q \bar{Q} \bar{Q}$ configuration, Eur. Phys. J. C 77, 709 (2017).

[8] V. Baru, C. Hanhart, and A. V. Nefediev, Can X(3915) be the tensor partner of the $X(3872)$ ?, J. High Energy Phys. 06 (2017) 010.
[9] F. E. Close and P. R. Page, The $D^{* 0} \bar{D}^{0}$ threshold resonance, Phys. Lett. B 578, 119 (2004).

[10] E. Braaten and M. Lu, Line shapes of the X(3872), Phys. Rev. D 76, 094028 (2007).

[11] S. Coito, G. Rupp, and E. van Beveren, $X(3872)$ is not a true molecule, Eur. Phys. J. C 73, 2351 (2013).

[12] X. Liu, Y.-R. Liu, W.-Z. Deng, and S.-L. Zhu, Is $Z^{+}(4430)$ a loosely bound molecular state?, Phys. Rev. D 77, 034003 (2008).

[13] C.-Y. Cui, X.-H. Liao, Y.-L. Liu, and M.-Q. Huang, Could $Z_{c}(3900)$ be a $I^{G} I^{P}=1^{+} 1^{+} D^{*} \bar{D}$ molecular state?, J. Phys. G 41, 075003 (2014).

[14] Q. Wang, C. Hanhart, and Q. Zhao, Decoding the Riddle of $Y(4260)$ and $Z_{c}(3900)$, Phys. Rev. Lett. 111, 132003 (2013).

[15] A. Esposito, A. Pilloni, and A. Polosa, Hybridized tetraquarks, Phys. Lett. B 758, 292 (2016).

[16] N. Isgur, R. Kokoski, and J. Paton, Gluonic Excitations of Mesons: Why They Are Missing and Where to Find Them, Phys. Rev. Lett. 54, 869 (1985).

[17] L. Liu, G. Moir, M. Peardon, S. M. Ryan, C. E. Thomas, P. Vilaseca, J. J. Dudek, R. G. Edwards, B. Joó, and D. G. Richards (for the Hadron Spectrum Collaboration), Excited 
and exotic charmonium spectroscopy from lattice QCD, J. High Energy Phys. 07 (2012) 126.

[18] G. Li, Hidden-charmonium decays of $Z_{c}(3900)$ and $Z_{c}(4025)$ in intermediate meson loops model, Eur. Phys. J. C 73, 2621 (2013).

[19] J.-R. Zhang, Improved QCD sum rule study of $Z_{c}(3900)$ as a $\bar{D} D^{*}$ molecular state, Phys. Rev. D 87, 116004 (2013).

[20] S. H. Blitz and R. F. Lebed, Tetraquark cusp effects from diquark pair production, Phys. Rev. D 91, 094025 (2015).

[21] E. S. Swanson, Cusps and exotic charmonia, Int. J. Mod. Phys. E 25, 1642010 (2016).

[22] P. Pakhlov and T. Uglov, Charged charmonium-like $Z^{+}$(4430) from rescattering in conventional $B$ decays, Phys. Lett. B 748, 183 (2015).

[23] F.-K. Guo, C. Hanhart, Q. Wang, and Q. Zhao, Could the near-threshold $X Y Z$ states be simply kinematic effects?, Phys. Rev. D 91, 051504 (2015).

[24] S. Dubynskiy and M. Voloshin, Hadro-charmonium, Phys. Lett. B 666, 344 (2008).

[25] X. Li and M. B. Voloshin, $Y(4260)$ and $Y(4360)$ as mixed hadrocharmonium, Mod. Phys. Lett. A 29, 1450060 (2014).

[26] M. Ablikim et al., Observation of a Charged Charmoniumlike Structure in $e^{+} e^{-} \rightarrow \pi^{+} \pi^{-} J / \psi$ at $\sqrt{s}=4.26 \mathrm{GeV}$, Phys. Rev. Lett. 110, 252001 (2013).

[27] Z. Q. Liu et al., Study of $e^{+} e^{-} \rightarrow \pi^{+} \pi^{-} J / \psi$ and Observation of a Charged Charmoniumlike State at Belle, Phys. Rev. Lett. 110, 252002 (2013).

[28] B. Aubert et al., Observation of a Broad Structure in the $\pi^{+} \pi^{-} J / \psi$ Mass Spectrum Around $4.26 \mathrm{GeV} / c^{2}$, Phys. Rev. Lett. 95, 142001 (2005).

[29] Q. He et al., Confirmation of the $Y(4260)$ resonance production in initial state radiation, Phys. Rev. D 74, 091104 (2006).

[30] C.Z. Yuan et al., Measurement of the $e^{+} e^{-} \rightarrow \pi^{+} \pi^{-} J / \psi$ Cross Section via Initial-State Radiation at Belle, Phys. Rev. Lett. 99, 182004 (2007).

[31] T. Xiao, S. Dobbs, A. Tomaradze, and K. K. Seth, Observation of the charged hadron $Z_{c}^{ \pm}(3900)$ and evidence for the neutral $Z_{c}^{0}(3900)$ in $e^{+} e^{-} \rightarrow \pi \pi J \psi$ at $\sqrt{s}=4170 \mathrm{MeV}$, Phys. Lett. B 727, 366 (2013).

[32] M. Ablikim et al., Observation of a Charged $\left(D \bar{D}^{*}\right)^{ \pm}$Mass Peak in $e^{+} e^{-} \rightarrow \pi D \bar{D}^{*}$ at $\sqrt{s}=4.26 \mathrm{GeV}$, Phys. Rev. Lett. 112, 022001 (2014).

[33] M. Ablikim et al., Observation of $Z_{c}(3900)^{0}$ in $e^{+} e^{-} \rightarrow \pi^{0} \pi^{0} J / \psi$, Phys. Rev. Lett. 115, 112003 (2015).

[34] M. Ablikim et al., Confirmation of a charged charmoniumlike state $Z_{c}(3885)^{\mp}$ in $e^{+} e^{-} \rightarrow \pi^{ \pm}\left(d \bar{d}^{*}\right)^{\mp}$ with double $d$ tag, Phys. Rev. D 92, 092006 (2015).

[35] M. Ablikim et al., Observation of a Neutral Structure Near the $D \bar{D}^{*}$ Mass Threshold in $e^{+} e^{-} \rightarrow\left(D \bar{D}^{*}\right)^{0} \pi^{0}$ at $\sqrt{s}=$ 4.226 and 4.257 GeV, Phys. Rev. Lett. 115, 222002 (2015).

[36] V. M. Abazov et al., Evidence for $Z_{c}^{ \pm}(3900)$ in semiinclusive decays of $b$-flavored hadrons, Phys. Rev. D 98, 052010 (2018).
[37] C. Liu, L. Liu, and K.-L. Zhang, Towards the understanding of $Z_{c}(3900)$ from lattice QCD, Phys. Rev. D 101, 054502 (2020).

[38] L.S. Kisslinger and S. Casper, $Z_{c}(3900)$ as a four-quark state, Int. J. Theor. Phys. 54, 3825 (2015).

[39] S. S. Agaev, K. Azizi, and H. Sundu, Strong $Z_{c}^{+}(3900) \rightarrow j / \psi \pi^{+} ; \eta_{c} \rho^{+}$decays in QCD, Phys. Rev. D 93, 074002 (2016).

[40] S. S. Agaev, K. Azizi, and H. Sundu, Treating $Z_{c}(3900)$ and $z(4430)$ as the ground state and first radially excited tetraquarks, Phys. Rev. D 96, 034026 (2017).

[41] W. Chen, T. G. Steele, H.-X. Chen, and S.-L. Zhu, Mass spectra of $Z_{c}$ and $Z_{b}$ exotic states as hadron molecules, Phys. Rev. D 92, 054002 (2015).

[42] Y. Dong, A. Faessler, T. Gutsche, and V. E. Lyubovitskij, Strong decays of molecular states $Z_{c}^{+}$and $Z_{c}^{\prime+}$, Phys. Rev. D 88, 014030 (2013).

[43] S. Patel, M. Shah, and P. C. Vinodkumar, Mass spectra of four-quark states in the hidden charm sector, Eur. Phys. J. A 50, 131 (2014).

[44] K. Azizi and N. Er, X(3872): Propagating in a dense medium, Nucl. Phys. B936, 151 (2018).

[45] T. Cohen, R. Furnstahl, D. Griegel, and X. Jin, QCD sum rules and applications to nuclear physics, Prog. Part. Nucl. Phys. 35, 221 (1995).

[46] R. J. Furnstahl, D. K. Griegel, and T. D. Cohen, QCD sum rules for nucleons in nuclear matter, Phys. Rev. C 46, 1507 (1992).

[47] B. Ioffe, QCD (Quantum chromodynamics) at low energies, Prog. Part. Nucl. Phys. 56, 232 (2006).

[48] X. Jin, T. D. Cohen, R. J. Furnstahl, and D. K. Griegel, QCD sum rules for nucleons in nuclear matter II, Phys. Rev. C 47, 2882 (1993).

[49] P. E. Shanahan, A. W. Thomas, and R. D. Young, Sigma terms from an SU(3) chiral extrapolation, Phys. Rev. D 87, 074503 (2013).

[50] N. Er and K. Azizi, Fate of the doubly heavy spin-3/2 baryons in a dense medium, Phys. Rev. D 99, 074012 (2019).

[51] K. Azizi and N. Er, Effects of a dense medium on parameters of doubly heavy baryons, Phys. Rev. D 100, 074004 (2019).

[52] A. Pilloni, C. Fernndez-Ramrez, A. Jackura, V. Mathieu, M. Mikhasenko, J. Nys, and A. Szczepaniak, Amplitude analysis and the nature of the $Z_{c}(3900)$, Phys. Lett. B 772, 200 (2017).

[53] Z.-G. Wang and T. Huang, Analysis of the $X(3872)$, $Z_{c}(3900)$, and $Z_{c}(3885)$ as axial-vector tetraquark states with QCD sum rules, Phys. Rev. D 89, 054019 (2014).

[54] Z.-G. Wang and T. Huang, Possible assignments of the $X(3872), Z_{c}(3900)$, and $Z_{b}(10610)$ as axial-vector molecular states, Eur. Phys. J. C 74, 2891 (2014).

[55] K. Kim, H. K. Lee, and J. Lee, Compact star matter: EoS with new scaling law, Int. J. Mod. Phys. E 26, 1740011 (2017). 